\title{
A qualitative exploratory study of selected physicians' perceptions of the management of non-communicable diseases at a referral hospital in Zimbabwe
}

\author{
Alexander Cheza ${ }^{*}$ (I) and Boikhutso Tlou
}

\begin{abstract}
Introduction: Non-communicable diseases (NCDs) have recently become a global public health burden and a leading cause of premature death, mainly in low- and middle-income countries (LMICS). The aim of the study was to explore physicians' perceptions on the availability and quality of clinical care for the management of NCDs.

Methods: This was a qualitative exploratory study meant to obtain expert perceptions on clinical care delivery for NCDs in one Zimbabwean central hospital setting. Data was collected from participants who consented and was analyzed using Stata version 13. A four-point Likert scale was used to categorize different levels of perceived satisfaction.

Findings: Twenty-three doctors participated in the study: four female doctors and nineteen males. Nineteen of the doctors were general practitioners, whilst four were specialists. The findings indicated that both categories perceived some shortfalls in clinical care for NCDs. Moreover, the perceptions of general practitioners and specialists were not significantly different. Participants perceived cancer care to be lagging far behind the other three NCDs under study. Care of cardiovascular diseases (CVDs) and diabetes showed mixed perceptions amongst participants, with positive perceptions almost equaling negative perceptions. Furthermore, hypertension was perceived to be clinically cared for better than the other NCDs under consideration. Reasons for the gaps in NCD clinical care were attributed by $33 \%$ of the participants to financial challenges; a further $27 \%$ to patient behavioral challenges; and $21 \%$ to communication challenges.

Conclusions: The article concludes that care delivery for the selected NCDs under study at CCH need to be improved. Furthermore, it is crucial to diagnose NCDs before patients show clinical symptoms. This helps disease prognosis to yield better care results. The evaluation of doctors' perceptions indicates the need to improve NCD care at the institution in order to control NCD co-morbidities that may increase mortality.
\end{abstract}

Keywords: Non-communicable diseases (NCDs), Healthcare, Cancers, Diabetes, Cardiovascular disease (CVD), Hypertension

\footnotetext{
* Correspondence: 218086530@stu.ukzn.ac.za

School of Nursing and Public Health, University of KwaZulu-Natal, 1st Floor, George Campbell Building, Howard College Campus, UKZN, Durban 4000, South Africa
}

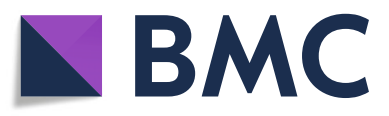

(c) The Author(s). 2021 Open Access This article is licensed under a Creative Commons Attribution 4.0 International License, which permits use, sharing, adaptation, distribution and reproduction in any medium or format, as long as you give appropriate credit to the original author(s) and the source, provide a link to the Creative Commons licence, and indicate if changes were made. The images or other third party material in this article are included in the article's Creative Commons licence, unless indicated otherwise in a credit line to the material. If material is not included in the article's Creative Commons licence and your intended use is not permitted by statutory regulation or exceeds the permitted use, you will need to obtain permission directly from the copyright holder. To view a copy of this licence, visit http://creativecommons.org/licenses/by/4.0/ The Creative Commons Public Domain Dedication waiver (http://creativecommons.org/publicdomain/zero/1.0/) applies to the data made available in this article, unless otherwise stated in a credit line to the data. 


\section{Introduction}

Non-communicable diseases (NCDs) have recently become a global public health burden, contributing more than half of the global health loss [1]. NCDs account for between 60 and $70 \%$ of all deaths globally [1, 2]. NCDs are also a leading cause of premature death, $80 \%$ of which are in low- and middle-income countries (LMICs) [1]. The World Health Organization (WHO) also reported a $71 \%$ increase in morbidity and mortality related to NCDs, with more than 40.5 million deaths from the total 56.9 million global deaths recorded in 2016 being related to NCDs, three quarters of which were in LMICs $[3,4]$. The NCD burden is exacerbated in LMICs due to co-morbidities with the HIV burden [5]. The WHO estimated that by 2020 , NCDs were expected to result in approximately $80 \%$ of the worldwide disease burden, causing about seven in every ten deaths in LMICs $[3,6]$.

Apart from the high mortality and morbidity of NCDs, healthcare systems in most LMICs are generally illprepared, fragile, under-resourced and face infrastructural limitations in dealing with the epidemiological and economic costs associated with NCDs [6-8]. This is evident through disparities in health statuses and lifeexpectancies between high-income countries and LMICs, particularly in relation to NCDs, which is attributable to poor healthcare delivery and management systems in LMICs [9]. A healthcare delivery system is defined as a combination of organized people, organizations and resources for the purposes of delivering healthcare services to meet the health needs of a target population [10]. Healthcare services may be delivered by single-provider practices or a big healthcare ecosystem. Moreover, healthcare systems must provide quality healthcare services, whereby the World Health Organization defines "quality as the degree to which health services for individuals and populations increase the likelihood of desired health outcomes and are consistent with current professional knowledge" [11]. Hence, the quality of healthcare services affects the level of satisfaction, which refers to the fulfilment of physicians' expectations in the healthcare delivery system for NCD patients.

It is apparent that LMICs need to improve the performance of their healthcare systems with regard to NCDs. Global efforts for the prevention and control of NCDs have intensified after the endorsement of the Global Strategy for the Prevention and Control of Non-communicable Diseases [12]. However, the effective prevention, control and management of NCDs requires properly planned healthcare systems and response mechanisms [11]. These response mechanisms must incorporate a multiplicity of stakeholders/sectors, including those not directly involved in health [13].
Literature records that NCDs are a major public health burden in the Sub-Saharan Africa (SSA) region, with significant morbidity and mortality [14]. A few studies have been conducted in Zimbabwe to assess the disease burden of selected NCDs, but the authors did not find literature evaluating the management and control of NCDs. For instance, Smit et al. (2015) examined the burden of NCDs in people living with HIV (PLHIV) in Zimbabwe [15]. The results showed that PLHIV had a $19.6 \%$ chance of being diagnosed with at least one NCD and a $4.6 \%$ likelihood of being diagnosed with more than one NCD [11]. Other studies focused on the prevalence of hypertension. Chimberengwa and Naidoo (2019) examined the knowledge, attitudes and practices related to hypertension in a rural setting in Zimbabwe [16]. Additionally, Chireshe and Naidoo (2019) studied hypertension amongst patients treated at Parirenyatwa Hospital, Zimbabwe's biggest referral hospital, and found an incidence of hypertension of $29.9 \%$ [17]. In a meta-analysis, Mutowo et al. (2015) found a hypertension prevalence of $30 \%$, with a higher burden in urban areas than in rural areas [18]. Overall, the WHO in Zimbabwe estimates that NCDs accounted for about $33 \%$ of the mortalities recorded in 2016 [19].

The management of NCDs in LMICs faces a myriad challenges such as poor laboratory facilities; frequent medicine stock-outs; a limited and poorly distributed health workforce and pharmaceuticals; and poor access to financial resources for caregivers and clients, amongst many other challenges $[20,21]$. The challenges highlighted above also affect the management and control of NCDs in Zimbabwe.

The improvement of the management and control of NCDs by the Ministry of Health and Child Care (MoHCC) in Zimbabwe, in cooperation with the WHO, has been a strategic priority since 2016 [22]. The management of NCDs in Zimbabwe includes several personnel who are either directly or indirectly involved in the daily management of different NCDs. These personnel include physicians, laboratory scientists, nurses, pharmacists, administrators and community health workers, amongst others. Physicians are amongst the primary direct caregivers with the responsibility for screening, diagnosis, ordering further examinations, reviewing and treatment of NCD patients. The authors elected to evaluate physicians' perceptions in order to gather expert opinions in light of the fact that physicians play a key role in healthcare, since the ultimate responsibility for patients' care rests on them. This study can be a foundation for expanding the scope of research to evaluate perceptions by other NCD-care personnel.

The aim was to conduct an exploratory study to evaluate the availability and quality of NCDs clinical care based on the perceptions of physicians who attend to 
NCD patients at Chitungwiza Central Hospital $(\mathrm{CCH})$, a referral hospital located in the Harare metropolitan province of Zimbabwe. The study was also motivated by the existence of scant literature on evaluations of care delivery systems for the management and control of NCDs by caregivers such as physicians in developing countries, including Zimbabwe.

\section{Materials and methods Study design}

This was a qualitative exploratory study meant to obtain expert perceptions of care delivery for NCDs in one Zimbabwean referral hospital setting. Data was collected from participants who consented and was analyzed using Stata version 13 .

\section{Study setting}

The study was conducted at $\mathrm{CCH}$, which is a referral hospital with a catchment area including urban, periurban and rural locations. There are about 15 hospitals in Mashonaland East Province surrounding $\mathrm{CCH}$. In addition, $\mathrm{CCH}$ serves an estimated population of 1.5 million people, spread over an estimated $32,230 \mathrm{~km}^{2}$ area. $\mathrm{CCH}$ attends to an average of $80 \mathrm{NCD}$ patients in the outpatients' department and 20 in the inpatients' department daily. The economic status of the population within the catchment area of the $\mathrm{CCH}$ is generally poor, with the majority living below the national poverty line, averaging US $\$ 220$ monthly for a family of five [23], a common feature for most settings in Zimbabwe due to high unemployment levels. The population is therefore mostly unemployed or self-employed, embarking on livelihood sustenance projects such as market gardening, vending and other informal economic activities.

\section{Recruitment of participants}

From a total population of 43 medical doctors engaged by $\mathrm{CCH}$ on a part-time or full-time basis, 26 doctors, including specialists, were recruited to participate in the study based on the inclusion/exclusion criteria provided below. Only doctors who consented to the study were included in the study, while doctors who do not provide clinical care for NCD-related conditions were excluded from participating in the study, For, example dental surgeons.

\section{i) Inclusion Criteria}

- Medical doctors employed by the $\mathrm{CCH}$, both on a full-time or part-time basis, who provide clinical care to patients with NCD-related conditions.
- Only doctors who consented to participate in the study were included.

\section{ii) Exclusion Criteria}

- Other hospital staff who are not medical doctors.

\section{Data collection procedures}

Data was collected from both sessional and resident doctors at $\mathrm{CCH}$ through a self-administered questionnaire. To ensure that there was minimum bias regarding the respondents' perceptions, the anonymity of the participants was assured in both data collection and analysis. The questionnaires were distributed electronically through the Survey Monkey platform. The participants included general practitioners and specialists providing clinical care for NCD-related conditions.

The selection of study participants was based on their knowledge and experience in the management of NCDs. Therefore, doctors who do not provide clinical care for NCDs or any related conditions were excluded from the study. All the participants who met the inclusion criteria were communicated with in order to obtain their consent and thereafter, a link to the online questionnaire was distributed via email to the 26 participants who consented.

In order to assess the quality of clinical care, the World Health Organization's definition of quality was used as the basis for assessing quality, that is, "the extent to which the healthcare services provided to individuals and patient populations improve desired health outcomes. To achieve this, healthcare must be safe, effective, timely, efficient, equitable and patient-centered" [10]. The quality of healthcare is a collaborative effort involving the patient, physician, patient's family and the community. Therefore, it can be assessed from the perspectives of any of these. Accordingly, physicians' perceptions of quality were assessed based on the features highlighted in the above definition, measured using a four-point Likert scale with the following options:

\begin{tabular}{llll}
\hline Not satisfactory & Somehow satisfactory & Satisfactory & Very satisfactory \\
1 & 2 & 3 & 4
\end{tabular}

The Likert scale was used to quantify the qualitative evaluations of physicians' perceptions of clinical care with the following meanings: Not satisfactory means that physicians perceived the clinical care to be unable to meet the healthcare service expectations; Somehow satisfactory means that physicians were indifferent about the NCD clinical care, that is, it was meeting doctors' expectations to a lesser extent; Satisfactory means that the physicians perceived the clinical care to be just 
meeting basic healthcare needs; and Very satisfactory means that the physicians perceived the clinical care to be world-class and going beyond expectations.

\section{Data analysis procedures}

Data was analysed using Thematic Analysis [24]. Since some of the questions were open-ended, open coding was done, capturing the frequency of the major themes which are presented in tables and bar graphs. Stata version 13 was used for the statistical analysis of data and to plot bar graphs. Qualitative data was open coded first and subsequently axial coded before presentation and analysis [22]. The analysis of data was guided by themes that emerged from the data and related responses were analyzed together [22].

\section{Ethical consideration}

Ethical approvals for the study were received from the Biomedical Research Ethics Committee of the University of KwaZulu-Natal (BE057/19) and the Medical Research Council of Zimbabwe (MRCZ/A/2441). Participants provided informed consent prior to data collection.

\section{Results}

Twenty-six participants consented to the study, 23 of which completed and uploaded their responses. These are valid for the study, giving a response rate of $88.5 \%$. Their demographic characteristics are presented in Table 1 below.

As shown in Tables 1, 17.4\% of the valid responses were from female doctors, whilst $82.6 \%$ were from male doctors. Although most of the participants were general practitioners $(82.6 \%)$, the doctors were well experienced in providing clinical care for NCDs, as shown in Fig. 1.

As shown in Fig. 1, all specialists had been offering clinical care to NCD patients for more than 5 years, whilst $71 \%$ of general practitioners had more than 5 years of clinical care experience for NCD patients. The doctors gave their perceptions of the quality of clinical care services rendered to NCD patients. These responses are presented hereunder, starting with Fig. 2 depicting the physicians' perceptions of diabetes care at $\mathrm{CCH}$.

Figure 2 represents doctors' perceptions of the quality of clinical care services for diabetes. The quality of clinical care services for diabetic patients at $\mathrm{CCH}$ was perceived by $44 \%$ of general practitioner doctors as being satisfactory, whilst a further $44 \%$ of the general practitioners viewed the quality as somewhat satisfactory. The remaining $12 \%$ regarded diabetes clinical care to be unsatisfactory. In addition, $50 \%$ of the specialists regarded clinical care to be satisfactory and $25 \%$ regarded diabetes clinical care to be somewhat satisfactory. However, $25 \%$ of the specialists perceived the clinical care services for diabetic patients as not satisfactory.

Figure 3 below presents a summary of the physicians' perceptions of clinical care for cardiovascular diseases (CVD).

The quality of clinical care services rendered to CVD patients was perceived by $50 \%$ of specialists and $50 \%$ of general practitioners as being somewhat satisfactory. A further $12 \%$ of general practitioners and $25 \%$ of specialists regarded the quality of clinical care to be satisfactory, whilst $38 \%$ of general practitioners and $25 \%$ of specialists viewed the quality of CVD clinical care as not satisfactory.

The physicians' perceptions of the quality of clinical care for the management of hypertension showed that $25 \%$ of general practitioners regarded the quality of clinical care services as very satisfactory. In addition, $50 \%$ of specialists and $44 \%$ of general practitioners perceived the quality of clinical care for hypertension to be satisfactory. Furthermore, 25\% of both specialists and general practitioners regarded the quality of clinical care to be somewhat satisfactory; whilst $25 \%$ of specialists and $6 \%$ of general practitioners regarded the quality of clinical care to be not satisfactory. These results are shown in Fig. 4.

Lastly, in relation to the management of cancers, the doctors evaluated the quality of clinical care and the results of their perceptions are presented in Fig. 5. A majority of both general practitioners (69\%) and specialists (50\%) indicated that the quality of clinical care was not satisfactory. In addition, $25 \%$ of specialists and 19\% of general practitioners perceived the quality of care as somewhat satisfactory, whilst a further $25 \%$ of specialists and $13 \%$ of general practitioners regarded the quality of cancer clinical care as satisfactory.

Given the perceptions of both general practitioners and specialist doctors of the quality of clinical care, it was important to ascertain the circumstances under which these physicians would request further tests and

Table 1 Demographic Characteristics of Respondents $(n=23)$

\begin{tabular}{llll}
\hline Features & Description & Frequency & Percentage (\%) \\
\hline Gender & Male & 19 & 82.6 \\
& Female & 4 & 17.4 \\
Doctors' specialization & Specialist & 4 & 17.4 \\
& General practitioner & 19 & 82.6 \\
\hline
\end{tabular}




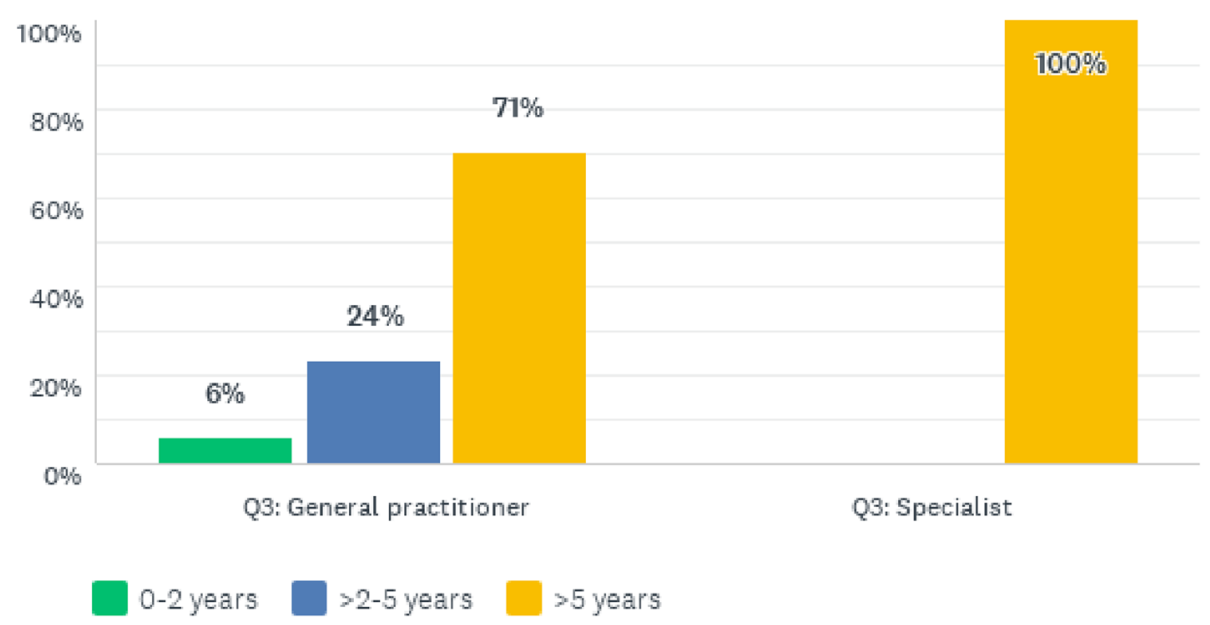

Fig. 1 Participants' experience offering clinical care to NCD patients

diagnosis. Screening and testing are critical for the effective management of NCDs since early detection makes most NCDs manageable and less fatal. The results are presented separately for the four NCDs under study. Figure 6 presents the reasons for both specialist and general practitioner doctors requesting testing for diabetic patients. It is observed that it is common practice for general practitioners (82\%) and specialists (75\%) to request further diabetes tests for patients presenting clinical symptoms. The rest of the reasons are shown in Fig. 6.

As presented in Fig. 7, it was also common practice to request screening tests for CVD patients presenting clinical signs and symptoms, as shown by $75 \%$ of specialists and $65 \%$ of general practitioners.
The predominant reasons for screening and testing for cancers, as shown in Fig. 8, are similar to CVDs and diabetes, as both specialists (100\%) and general practitioners (71\%) mainly requested screening for patients presenting with clinical signs and symptoms.

Of the four NCDs under study, hypertension presented a dissimilar predominant reason for screening and testing whereby almost all patients had their blood pressure checked when being attended to as part of the admission procedure. The reasons for testing for hypertension are shown in Fig. 9.

After establishing the conditions under which doctors requested further screening tests for effective management, the challenges that doctors faced when providing clinical care to NCD patients were enquired

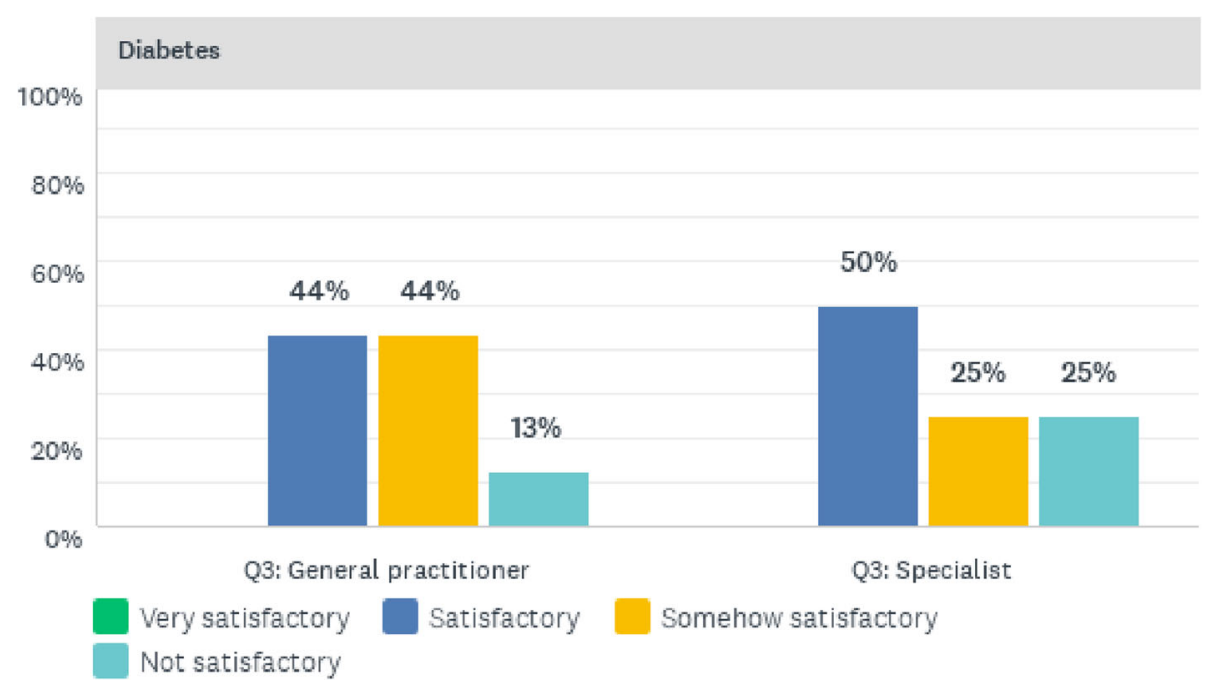

Fig. 2 Quality of diabetes clinical care services offered at $\mathrm{CCH}$ 


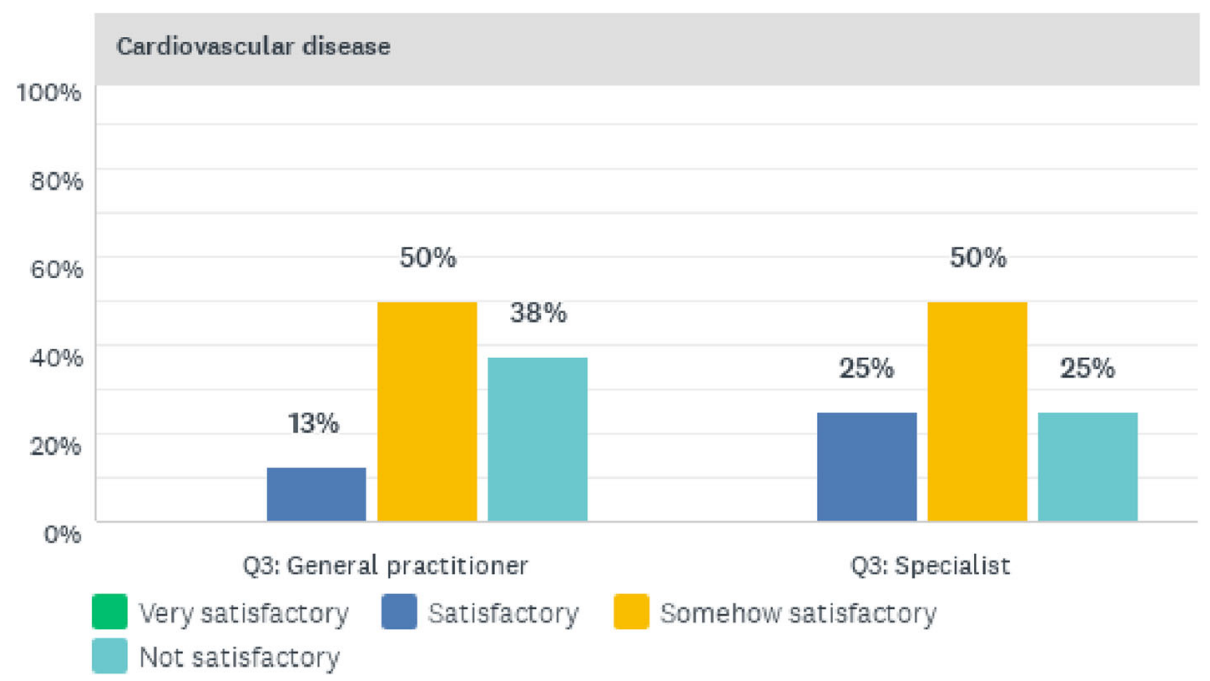

Fig. 3 Quality of CVD clinical care services offered at $\mathrm{CCH}$

from the doctors in order to establish their perspectives. The responses obtained in relation to these challenges are grouped by themes into related challenges and are summarized in Table 2 below.

Table 2 shows the five categories of challenges identified by the study, namely financial challenges, patient behavior challenges, supply chain challenges, communication challenges, as well as care provider challenges. The statistics regarding the highlighted challenges are provided in Fig. 10.

The most cited challenges were financial challenges, as perceived by $33 \%$ of the doctors. This was followed by patient behavioral challenges, which was cited by $27 \%$ of the doctors at $\mathrm{CCH}$. Another $21 \%$ of the doctors viewed communication as a challenge in the provision of care for NCD patients, whilst a further $10 \%$ cited challenges in the medical supply chain and $9 \%$ cited challenges with care providers for NCD care.

Given the challenges presented in Table 2 and summarized in Fig. 10, the study enquired about the gaps that the doctors considered to be stumbling blocks in the provision of care for NCD patients. The gaps established are summarized in Fig. 11 below.

The gap cited by the highest number of respondents was poverty and economic instability, as $20 \%$ of the doctors identified this factor as affecting the delivery of care to patients. The least identified gap was the varied methods used by doctors to manage different NCD patients, as cited by only $2 \%$ of the doctors. This shows a need for a standard framework for the screening and management of NCDs. Possible mitigatory measures

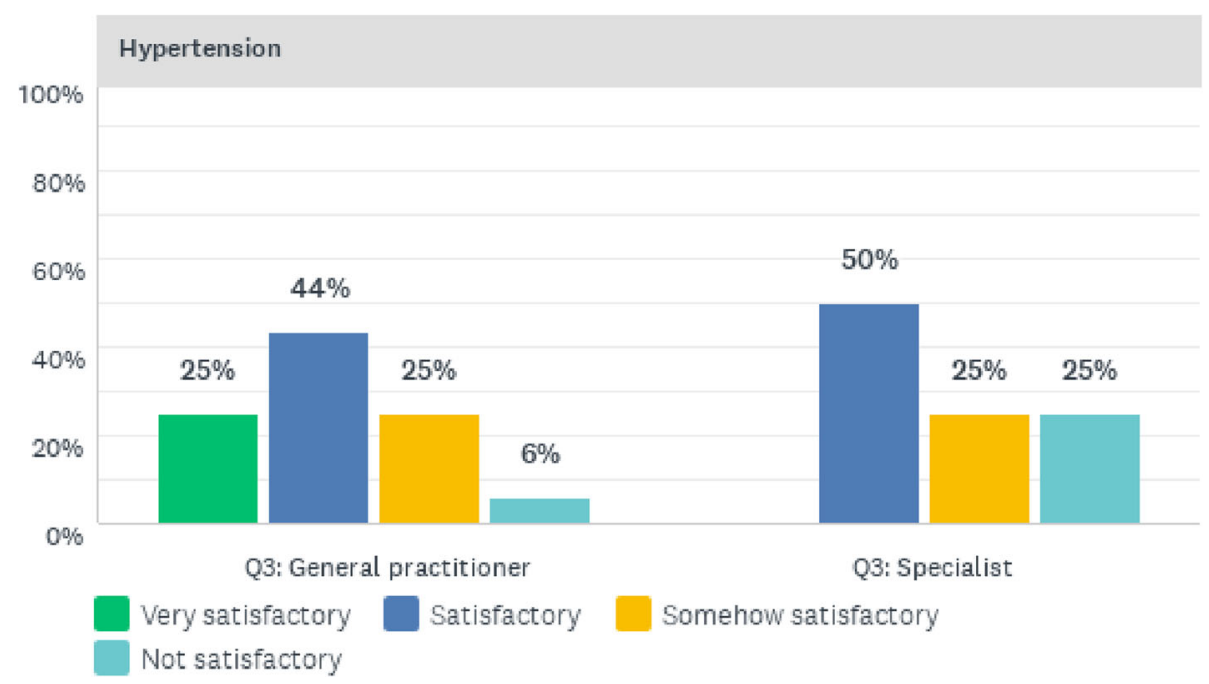

Fig. 4 Quality of hypertension clinical care services offered at $\mathrm{CCH}$ 


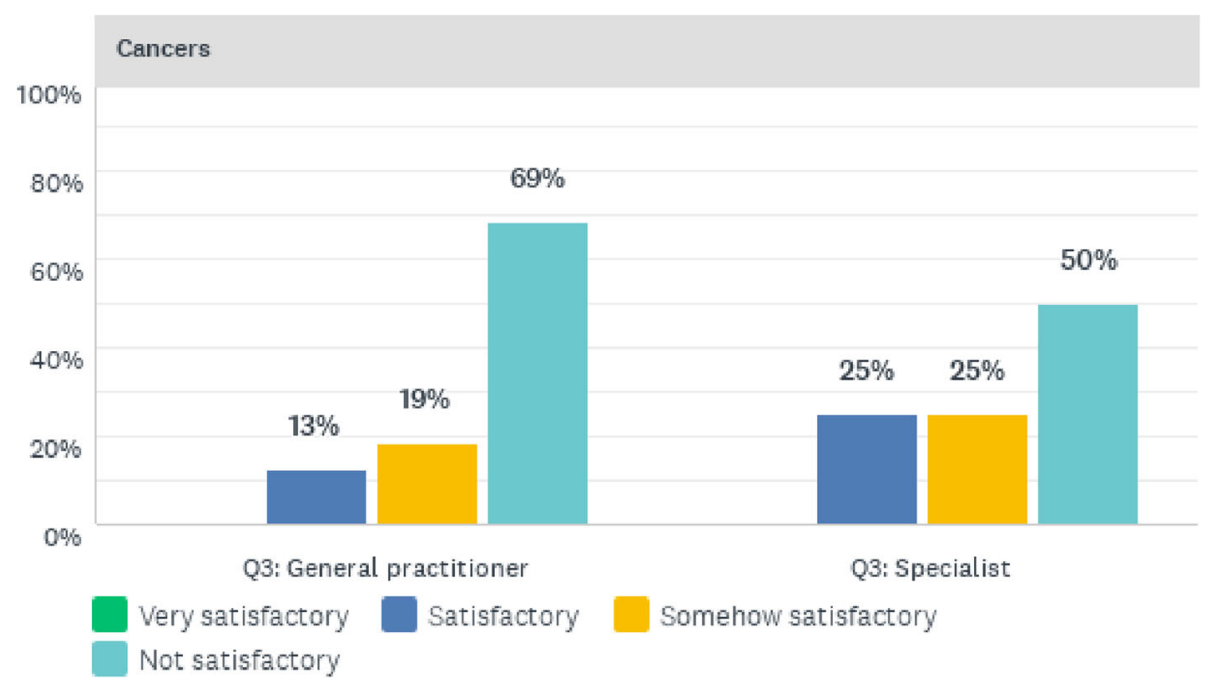

Fig. 5 Quality of cancer clinical care services offered at CCH

which could be implemented to improve the situation are summarized in Table 3 below.

As shown in Table 3, several possible measures are highlighted which could be implemented at $\mathrm{CCH}$ in order to mitigate the extent of NCDs and improve care delivery for NCD patients. According to $14 \%$ of the doctors, educational campaigns could help in mitigating the exposure of patients to various NCDs. A further $12 \%$ viewed the provision of funding from central government and development agencies as an effective strategy to mitigate the challenges faced in providing effective care to NCD patients. The strategies presented in Table 3 would be helpful in dealing with NCDs at public health institutions in Zimbabwe. The effectiveness of these strategies was not covered by this study and may require further enquiry.

\section{Discussion}

The aim of the study was to evaluate the availability and quality of NCDs clinical care based on the perceptions of physicians who attend to NCD patients at Chitungwiza Central Hospital (CCH), a referral hospital located in the Harare metropolitan province of Zimbabwe. The findings indicated perceived gaps in care delivery for $\mathrm{NCD}$ patients attended to at $\mathrm{CCH}$. The authors found that a significant number of the doctors thus viewed the quality of the care services offered to NCD patients to be unsatisfactory overall. This was

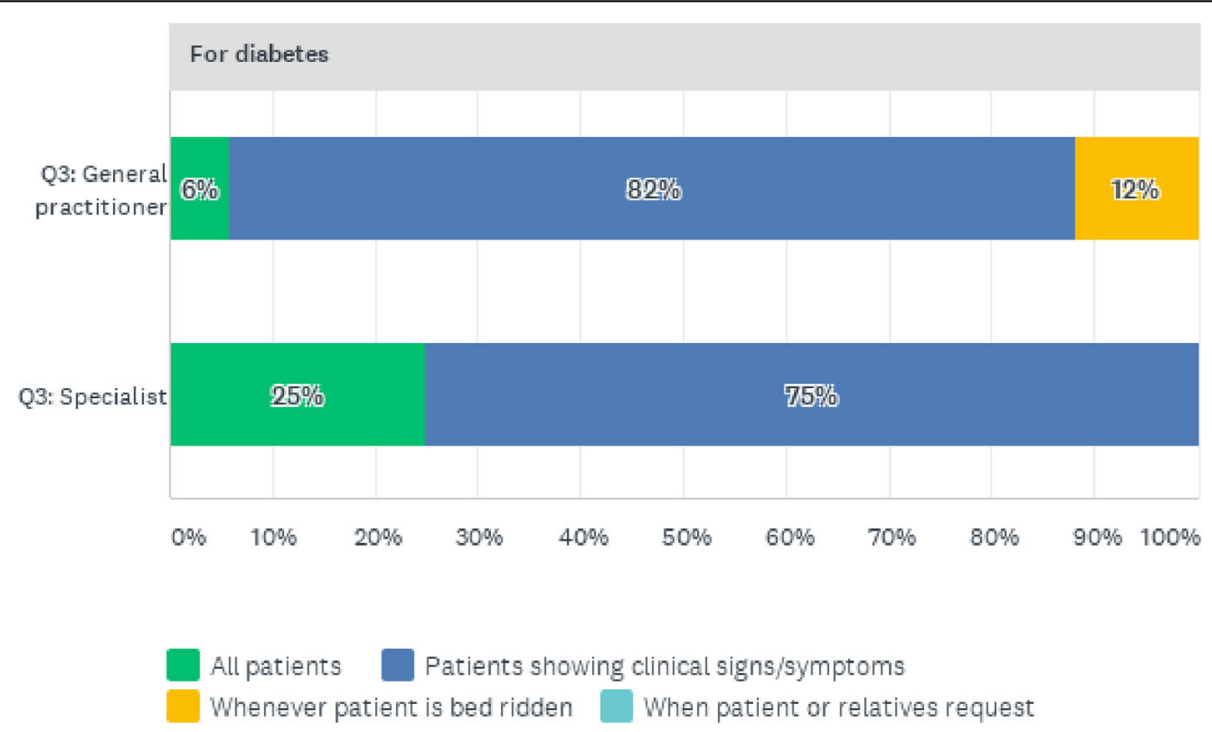

Fig. 6 Reasons for requesting Diabetes Screening Tests for Patients 


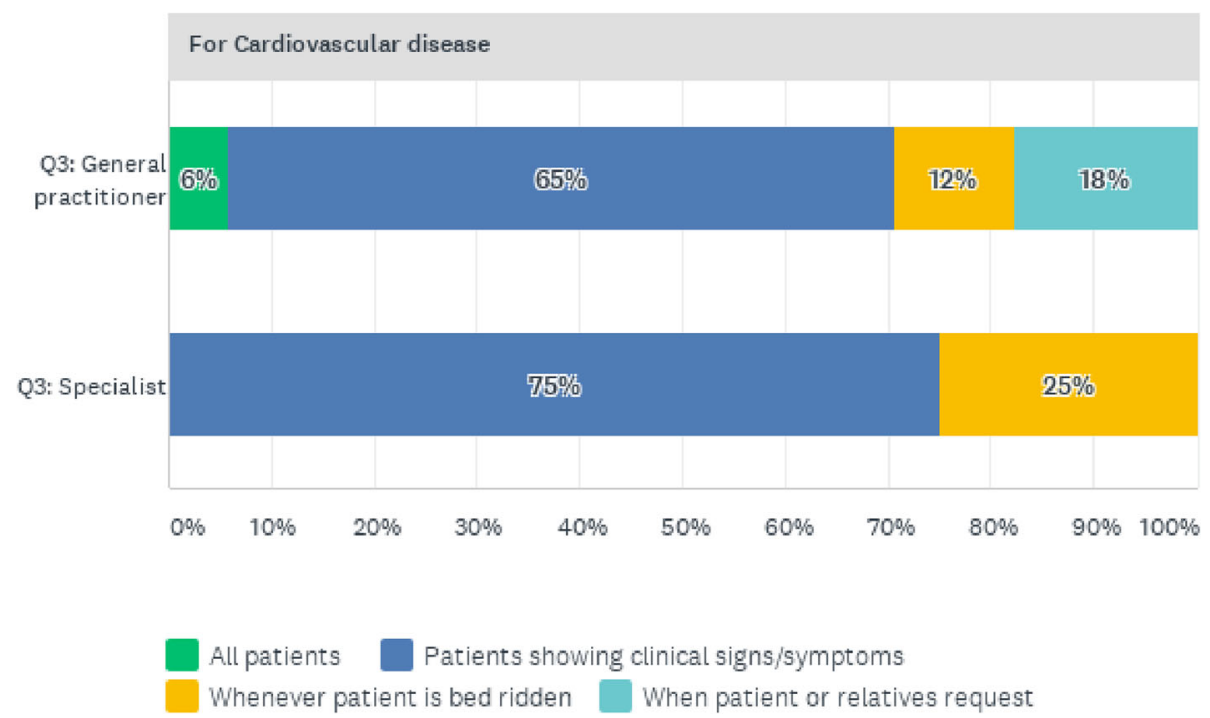

Fig. 7 Reasons for requesting CVD Screening tests for patients

attributable to various challenges impeding the smooth flow of care delivery for the selected NCDs.

The study also assessed the screening procedures for diabetes, hypertension, CVD and cancers. The authors observed that except for hypertension, which was screened for the majority of patients, the doctors first checked clinical signs and symptoms in order to request further tests. This is a matter of concern since NCDs may take time to be evident clinically, and in most cases will show signs at advanced stages, leading to poor prognosis of the condition. Devi, et al. elaborate further on the necessity of including screening and diagnosis of NCDs in the models for NCD management [8]. The study also identified the challenges faced by doctors in the management of NCDs. The most prevalent challenges were financial where the patients could not afford some of the procedures and services ordered by the physicians to manage the condition for better prognosis. The least challenging obstacle related to service provider challenges, since the study setting was in an urban location which is not badly affected by human resources challenges, which are common impediments in rural areas [25]. However, the researchers could not rule out possible bias since the participants were caregivers.

Doctors who participated in the study identified several gaps in clinical care delivery for NCD patients at $\mathrm{CCH}$ and proposed strategies that could be pursued in

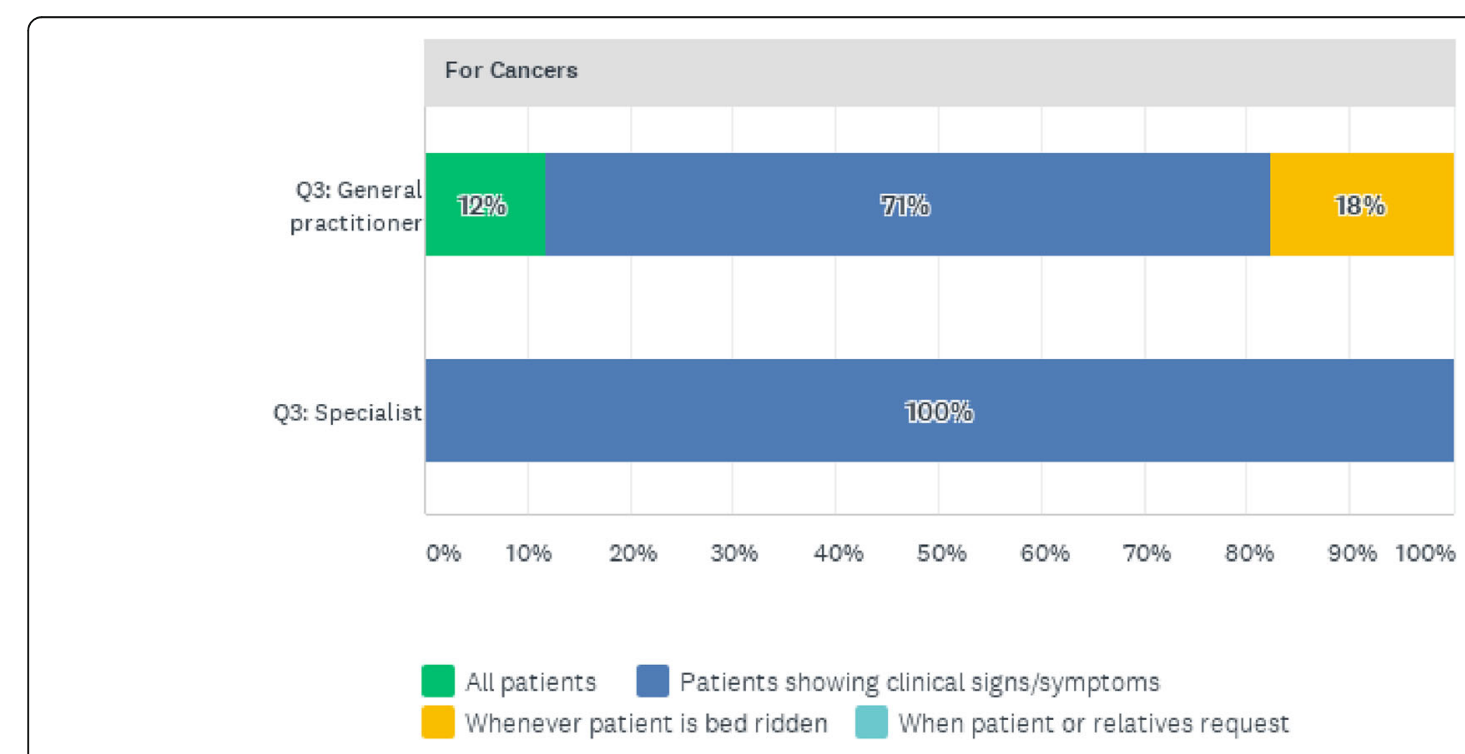

Fig. 8 Reasons for requesting Cancer Screening Tests for Patients 


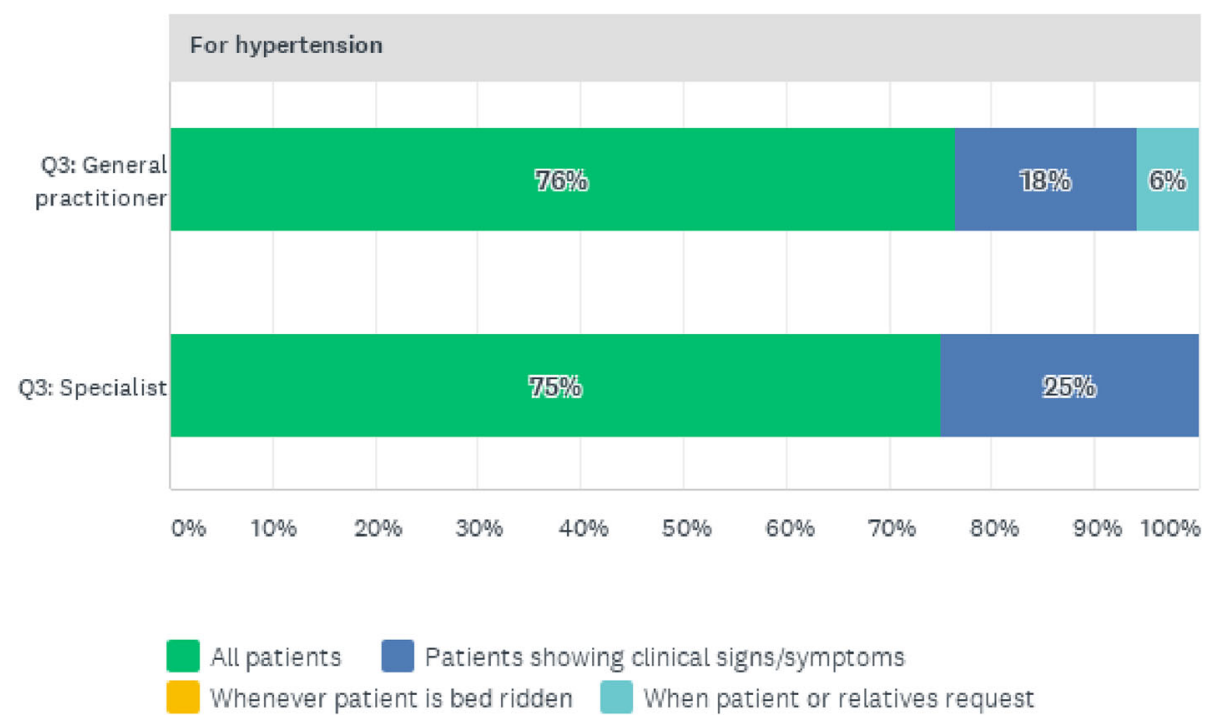

Fig. 9 Reasons for Hypertension Screening Tests for Patients

order to improve the quality of clinical care. Some of the proposed strategies include pursuing hospital-based tutoring to educate the community on the risk factors, disease burden and mortality associated with NCDs. $\mathrm{CCH}$ management and the responsible ministry should consider providing mandatory and free testing for NCDs for all patients in order to ensure early detection. This enhances the quality and effectiveness of the care and management of NCDs. Medical institutions such as $\mathrm{CCH}$ can improve clinical care quality through availing resources, medication and equipment for NCD screening and management at primary healthcare institutions.

The doctors' perceived unsatisfactory quality of clinical care delivery for NCD patients was consistent with findings obtained from other studies in almost similar settings, especially those in the SSA region $[2,6$,
7]. Poverty has been cited as a common cause for high NCD prevalence and the unsatisfactory care of NCD patients, as well as for the high mortality rates for various NCDs in the SSA region and LMICs $[3,5,8]$. This is attributable to various reasons, which include incapacitation to meet hospital bills for general medical check-ups, which are key to the early diagnosis of NCDs $[5,8)$. Moreover, poverty compromises educational achievements, which impedes the knowledge base on prevention and the lifestyle changes necessary to minimize exposure to NCDs $[3,25)$. The chronic nature of NCDs makes most of the populations from LMICs unable to afford the lifelong treatment required because of poverty [2].

Challenges faced in the care delivery for NCD patients were not unique to doctors at $\mathrm{CCH}$. Due to economic

Table 2 Challenges faced by Doctors providing quality NCD clinical care at CCH

\section{Financial Challenges}

No money for laboratory tests.

Unavailability of affordable screening services.

Drugs are unaffordable.

Lack of funding from government.

\section{Supply Chain Challenges}

Shortages of screening tools and equipment for tests.

Unavailability of drugs.

Shortages of advanced equipment.

\section{Care provider challenges}

No special testing laboratories.

Shortages of specialists.

Lack of expertise to support the patients.

\section{Patient Behavioral Challenges}

Non-compliance with lifestyle changes and denial.

Non-compliance with treatment.

Religious and Traditional beliefs which cause bad health-seeking behavior. Lack of co-operation from patients.

\section{Communication Challenges}

No clear guidelines for NCD patients.

Poor information dissemination.

A lack of basic knowledge about risk factors by the populace. 


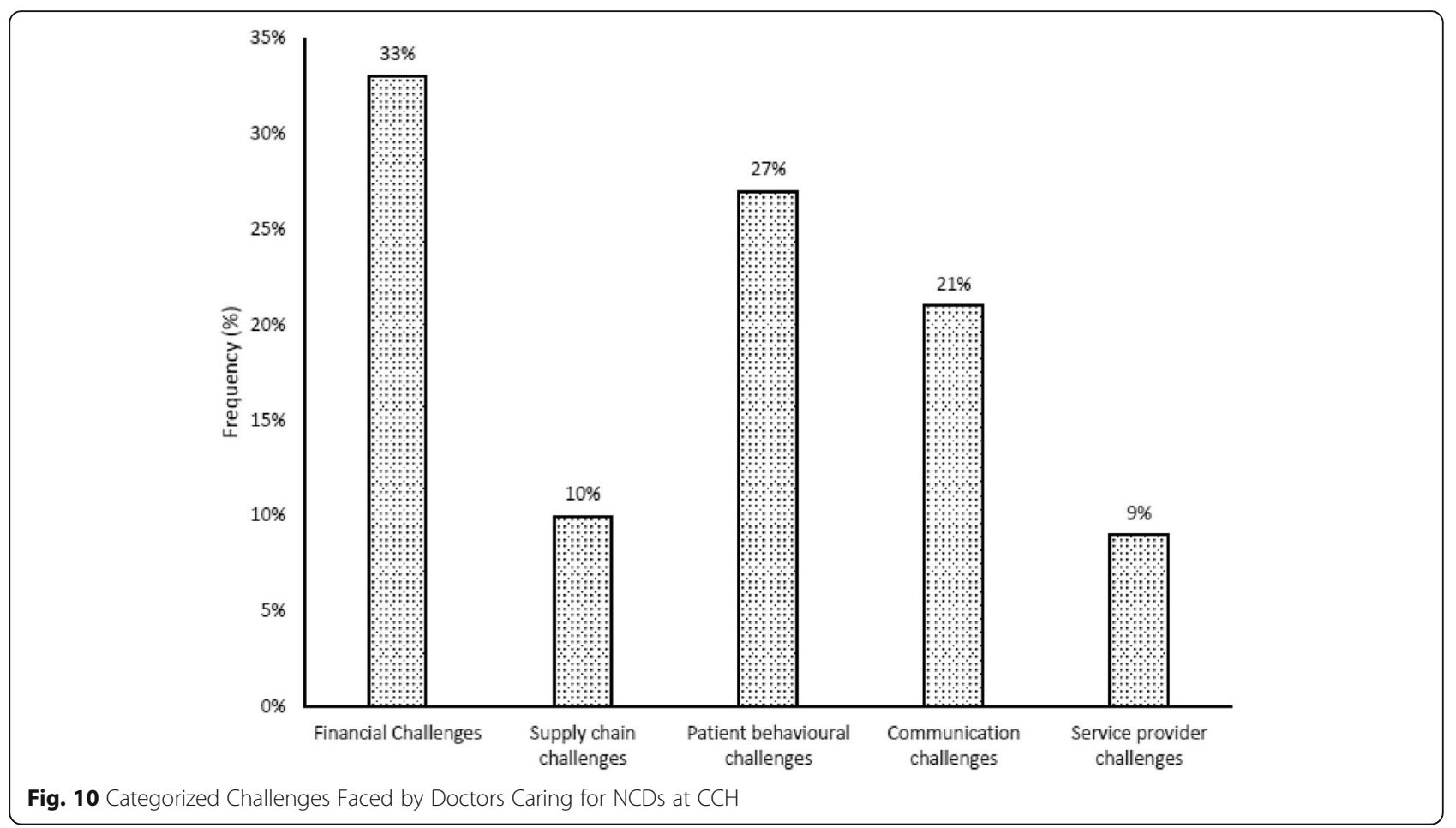

challenges in developing countries, financial challenges are common for NCD care providers in developing countries and the SSA region $[2,7,8]$. Doctors also highlighted perceived challenges related to patients' behavior, which exposes them to higher NCD risks [4]. Behaviors identified in literature include absconding from treatment due to stigma or the inability to afford medication, as well as religious practices such as faithhealing beliefs resulting in medication abscondment [4, $5,8]$. The study also revealed doctors' perceptions of the existence of behaviors by patients, which compromise care delivery for NCDs $[6,10]$. Patient behaviors and attitudes are common challenges in medicine whereby adherence to prescriptions is neglected and patients are sometimes unwilling to change behaviors such as smoking and alcohol abuse, despite the resultant exposure to undesirable effects such as NCD development $[4,5]$.

The strength of this exploratory study was primarily its contribution to an initial evaluation of NCD patients' care delivery from a referral hospital in a developing

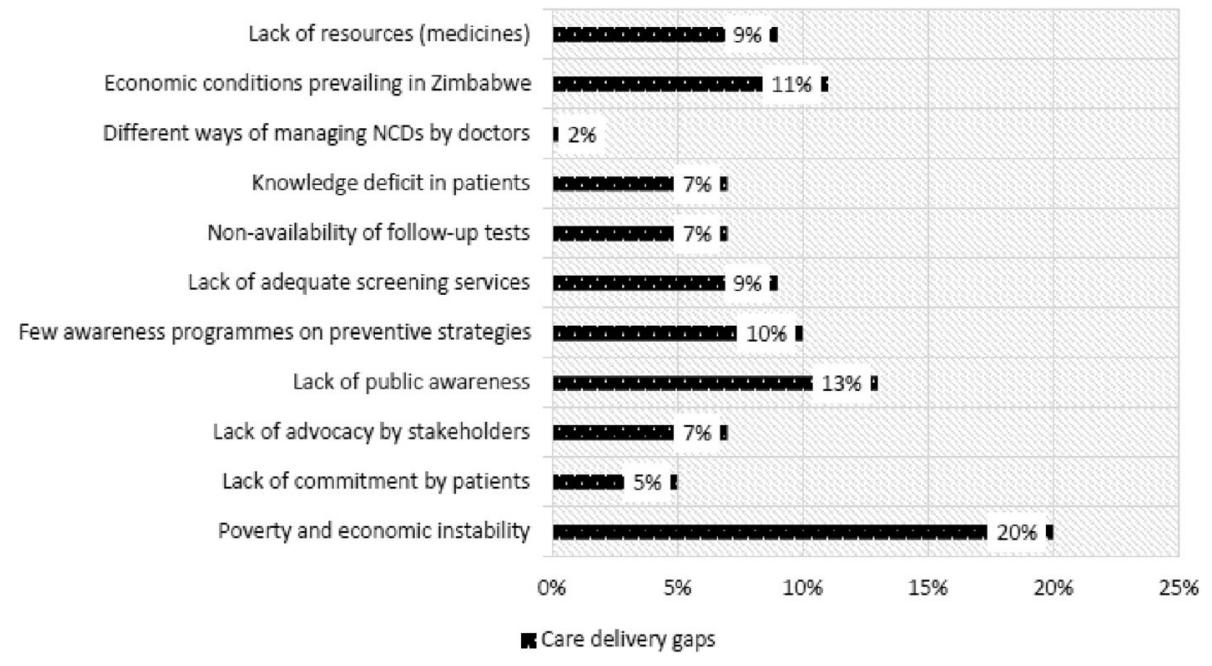

Fig. 11 Care Delivery Gaps 
Table 3 Mitigatory Measures to Close the Care Delivery Gaps for NCDs Patients

\begin{tabular}{ll}
\hline Possible mitigatory measures that could be implemented & Percentage (\%) \\
\hline Mandatory hospital-based tutoring for the public & 7 \\
Educational awareness campaigns & 14 \\
Better information dissemination through the media & 7 \\
A policy that speaks to NCDs & 7 \\
Free treatment at designated hospitals & 10 \\
Integration of health provision & 6 \\
Decentralization of NCD clinics to district hospitals & 4 \\
Screening tests at each clinic visit & 5 \\
Availing of funding from central government and development partners & 12 \\
Set up specific NCD clinics and help support them by providing adequate stocks of medication and laboratory equipment support & 3 \\
Affordable health insurance schemes & 10 \\
Give flexible timetables for patients' visits & 7 \\
Procure medicines on time & 7
\end{tabular}

country setting. The study population comprised medical doctors who are experts with varied experience in the management of NCDs. The study thus contributed towards the body of knowledge on the management of NCDs from an expert viewpoint. This research presents a baseline study that can guide further studies on evaluating NCD care delivery using either an expanded population from $\mathrm{CCH}$ or incorporating other care-givers in order to ascertain gaps and strategies for enhancing care delivery.

However, the main limitation of the study was the limited geographical coverage and the small sample used, which meant that the findings obtained in the study were not generalizable to be representative of the entire country and its health professionals. Moreover, there is a possibility of bias by respondents since the study was a self-evaluation. Therefore, doctors were unlikely to highlight their own weaknesses in NCD clinical care provision. The exclusion of patient observations was another limitation of the study. However, these limitations cannot invalidate the findings, since the study was exploratory in nature and hence there is need for conducting a study with a bigger population and sample.

The authors recommend that the management at $\mathrm{CCH}$ consider a deliberate policy for enhancing the quality of clinical care provided to NCD patients through the development and implementation of a service level agreement. Since $\mathrm{CCH}$ is a provincial hospital, the proposed policy can be disseminated to district clinics and hospitals. It is also recommended that all medical staff such as nurses be capacitated to offer acceptable quality NCD care to patients. Moreover, clinical processes must be re-engineered to ensure that $\mathrm{NCD}$ care is an integral part of patient care procedures at the institution. These recommendations are envisaged as having the ability to improve the quality of NCD clinical care at $\mathrm{CCH}$.

\section{Conclusions}

The study's findings showed that the clinical care offered for most NCDs at $\mathrm{CCH}$ is not of the quality and standard expected by the physicians to reduce morbidity and mortality. The quality of clinical care is compromised by challenges affecting healthcare institutions such as $\mathrm{CCH}$. There is an unavailability of sufficient equipment and/or affordable services for NCD care. Medical supplies are also limited in terms of availability and affordability. In addition, physicians at $\mathrm{CCH}$ perceived the existence of informational and knowledge gaps in the care and management of NCDs by patients. Doctors also observed that NCD patients fail to adhere to treatment protocols due to religious and traditional beliefs.

Based on the findings obtained in the study, the following conclusions were drawn regarding doctors' perceptions of care delivery for $\mathrm{NCD}$ patients at $\mathrm{CCH}$ :

a) Care delivery for NCD patients is perceived by some doctors as being unsatisfactory in meeting the requirements of the patients since there were challenges impeding their delivery of clinical care to NCD patients.

b) There are no clear guidelines and policies for healthcare practitioners on clinical care delivery for NCD patients. As a result, doctors do not have benchmarks for care delivery, as was evidenced by the varied responses from the doctors regarding the care of patients. 
c) There are no standard protocols for screening and managing NCDs, which encourages the early diagnosis of NCDs. This is likely to improve the quality of care, resulting in care which is safe, effective, timely, efficient, equitable and patientcentered.

The study is important in giving direction to both doctors and other stakeholders at $\mathrm{CCH}$ and in Zimbabwe on areas of possible improvement in NCD care delivery. The evaluation of doctors' perceptions of $\mathrm{NCD}$ clinical care delivery at $\mathrm{CCH}$ enlightens hospital management and policy-makers on the need to improve NCD care at the institution in order to control NCD comorbidities that may increase mortality. Findings from the study can also inform other low-income countries facing an escalation of NCDs burden and need to improve their clinical care delivery for persons diagnosed with NCDs. The study showed a need to improve timely NCD diagnosis, and the earlier commencement of care will ultimately give better results for the patient management. Policy makers, hospital management, and clinical caregivers in comparable settings can draw valuable lessons on ways to improve NCDs management and customize the findings to their unique clinical care settings.

\section{Abbreviations}

AIDS: Acquired Immune Deficiency Syndrome; $\mathrm{CCH}$ : Chitungwiza Central Hospital; CVD: Cardiovascular disease; HIV: Human Immunodeficiency Virus; LMICs: Low to Medium Income Countries; NCDs: Non-Communicable Diseases; Ol: Opportunistic Infections; PLHIV: People Living with Human Immunodeficiency Virus; SSA: Sub-Saharan Africa

\section{Supplementary Information}

The online version contains supplementary material available at https://doi. org/10.1186/s12992-021-00730-3.

Additional file 1. Questionnaire

Additional file 2. Anonymized Study Dataset.

\section{Acknowledgements}

We acknowledge the Ministry of Health and Child Care (Zimbabwe) and the $\mathrm{CCH}$ Administration Staff for the approval and assistance during the data collection. In addition, special gratitude goes to the University of Kwa-Zulu Natal's College of Health Sciences for all the support throughout the process.

\section{Authors' contributions}

AC, the Principal Investigator responsible for providing overall direction and oversight of the study at all stages fully engaged from the project design, protocol development and obtaining of ethical approvals to the study conduct, data analyses and dissemination of the study outcome. TB, Supervisor and Co-Investigator, responsible for technical direction, supervised and provided advice for all steps from the project design, protocol development and obtaining of ethical approvals to the study conduct, data analyses and dissemination of the study outcome. Reviewed the final article. The authors read and approved the final manuscript.

\section{Funding}

The study has not received external funding.

\section{Availability of data and materials}

The data that support the findings of this study are available and an anonymized dataset is included as an additional supporting file.

\section{Declarations}

Ethics approval and consent to participate

The Biomedical Research Ethics Committee of the University of Kwa-Zulu Natal (BE057/19) and the Medical Research Council of Zimbabwe (MRCZ/A/ 2441) granted ethical approvals. Participants provided informed consent prior to data collection.

\section{Consent for publication}

Written consent to participate in the study was a pre-requisite from each participant. Participants consented to the publication of the study findings without any jeopardy to their image.

\section{Competing interests}

The authors declare that they have no competing interests.

Received: 6 January 2021 Accepted: 7 July 2021

Published online: 19 July 2021

\section{References}

1. Benziger $C P$, Roth GA, Moran AE. The global burden of disease study and the preventable burden of NCD. Glob Heart. 2016;11(4):393-7. https://doi. org/10.1016/j.gheart.2016.10.024.

2. Unwin N, Samuels TA, Hassell T, Brownson RC, Guell C. The development of public policies to address non-communicable diseases in the Caribbean country of Barbados: the importance of problem framing and policy entrepreneurs. Int J Health Policy Manag. 2017;6(2):71-82. https://doi.org/1 0.15171/ijhpm.2016.74

3. World Health Organization (2017). Global Health Observatory (GHO) data: NCD mortality and morbidity. Available at: https://www.who.int/gho/ncd/ mortality_morbidity/en/ [Accessed 4 August 2020].

4. Rawal LB, Kharel C, Yadav UN, Kanda K, Biswas T, Vandelanotte C, et al. Community health workers for non-communicable disease prevention and control in Nepal: a qualitative study. BMJ Open. 2020;10(12):e040350. https://doi.org/10.1136/bmjopen-2020-040350.

5. Zungu NP, Mabaso ML, Kumalo F, Sigida S, Mlangeni L, Wabiri N, et al. Prevalence of non-communicable diseases (NCDs) and associated factors among HIV positive educators: findings from the 2015/6 survey of health of educators in public schools in South Africa. PLoS One. 2019;14(2):e0209756. https://doi.org/10.1371/journal.pone.0209756.

6. Islam SMS, Purnat TD, Phuong NTA, Mwingira U, Schacht K, Frösch G. Noncommunicable diseases (NCDs) in developing countries: a symposium report. Glob Health. 2014;10(81):1. https://doi.org/10.1186/s12992-014-0081-9.

7. Juma PA, Mohamed SF, Wisdom J, Kyobutungi C, Oti S. Analysis of noncommunicable disease prevention policies in five sub-Saharan African countries: study protocol. Arch Public Health. 2016;74(25):25. https://doi. org/10.1186/s13690-016-0137-9.

8. Devi R, Sehmi K, Kanitkar K, et al. A narrative review of the patient journey through the Lens of non-communicable diseases in low and middleincome countries. Adv Ther. 2020;37(12):4808-30. https://doi.org/10.1007/ s12325-020-01519-3.

9. Yamada T, Chen CC, Chiu IM, Rizvi SW. Non-communicable diseases in developing countries: causes and health policy/program assessments. J Trop Dis. 2013;1(117):1. https://doi.org/10.4172/2329-891X.1000117.

10. Piña IL, Cohen PD, Larson DB, Marion LN, Sills MR, Solberg LI, et al. A framework for describing health care delivery organizations and systems. Am J Public Health. 2015;105(4):670-9. https://doi.org/10.2105/AJPH.2014.3 01926.

11. World Health Organization. Handbook for national quality policy and strategy - a practical approach for developing policy and strategy to improve quality of care. Geneva: World Health Organization; 2018.

12. World Health Organization. Action plan for the global strategy for the prevention and control of noncommunicable diseases. Geneva: World Health Organization; 2008-2013.

13. Yaya S, Reddy KS, Belizán JM, Pingray V. Non-communicable diseases and reproductive health in sub-Saharan Africa: bridging the policy- 
implementation gaps. Reprod Health. 2020;17:8. https://doi.org/10.1186/s12 978-020-0857-8.

14. Mudie K, Tan MMJ, Kendall L, Addo J, Dos-Santos-Silva I, et al. Noncommunicable diseases in sub-Saharan Africa: a scoping review of large cohort studies. J Glob Health. 2019;9(2):020409. https://doi.org/10.7189/jogh. 09.020409 .

15. Smit M, Brinkman K, Geerlings S, Smit C, Thyagarajan K, Sighem Av, et al. Future challenges for clinical care of an ageing population infected with HIV: a modelling study [published correction appears in lancet infect dis. 2015 Sep;15(9):998]. Lancet Infect Dis. 2015;15(7):810-8. https://doi.org/10.1 016/S1473-3099(15)00056-0.

16. Chimberengwa PT, Naidoo M, on behalf of the cooperative inquiry group. Knowledge, attitudes and practices related to hypertension among residents of a disadvantaged rural community in southern Zimbabwe. PLoS One. 2019;14(6):e0215500. https://doi.org/10.1371/journal.pone.0215500.

17. Chireshe R, Naidoo K. Hypertension among human immunodeficiency virus infected patients on treatment at Parirenyatwa hospital: a descriptive study. Afr J Prim Health Care Fam Med. 2019;11(1):a1974. https://doi.org/10.4102/ phcfm.v11i1.1974.

18. Mutowo MP, Mangwiro JC, Lorgelly P, Owen A, Renzaho AM. Hypertension in Zimbabwe: a meta-analysis to quantify its burden and policy implications. World J Meta-Anal. 2015;3(1):54-60. https://doi.org/10.13105/wjma.v3.11.54.

19. World Health Organization. 2018. Noncommunicable Diseases (NCD) Country Profiles. Available at: https:/www.who.int/nmh/countries/zwe_en. pdf. [Accessed 28 March 2020].

20. Elias MA, Pati MK, Aivalli P, Srinath B, Munegowda C, Shroff ZC, et al. Preparedness for delivering non-communicable disease services in primary care: access to medicines for diabetes and hypertension in a district in South India. BMJ Glob Health. 2018;2(Suppl 3):e000519. https://doi.org/1 0.1136/bmjgh-2017-000519.

21. Witter S, Zou G, Diaconu K, Senesi RGB, Idriss A, et al. Opportunities and challenges for delivering non-communicable disease management and services in fragile and post-conflict settings: perceptions of policy-makers and health providers in Sierra Leone. BMC Conflict Health. 2020;14:3. https:// doi.org/10.1186/s13031-019-0248-3.

22. World Health Organization. 2018. Noncommunicable Diseases (NCD) Country Profiles. Available at: ccsbrief_zwe_en.pdf; jsessionid=DF939C4 7242119C538D330EOBCOB5C26(who.int). [Accessed: 6 Mar 2021].

23. The Chronicle 2020. Poverty Datum Line jumps 4,1pc to ZWD\$17 957. Available at: Poverty Datum Line jumps 4,1pc to \$17 957| The Chronicle [Accessed: 21 Dec 2020].

24. Vollstedt M, Rezat S. An introduction to grounded theory with a special focus on axial coding and the coding paradigm. In: Kaiser G, Presmeg N, editors. Compendium for early career researchers in mathematics education. ICME-13 monographs. Cham: Springer; 2019.

25. Mangundu M, Roets L, Janse van Rensberg E. Accessibility of healthcare in rural Zimbabwe: The perspective of nurses and healthcare users. Afr J Prm Health Care Fam Med. 2020;12(1):a2245. https://doi.org/10.4102/phcfm.v12 i1.2245.

\section{Publisher's Note}

Springer Nature remains neutral with regard to jurisdictional claims in published maps and institutional affiliations.

Ready to submit your research? Choose BMC and benefit from:
- fast, convenient online submission
- thorough peer review by experienced researchers in your field
- rapid publication on acceptance
- support for research data, including large and complex data types
- gold Open Access which fosters wider collaboration and increased citations
- maximum visibility for your research: over 100M website views per year
At BMC, research is always in progress.
Learn more biomedcentral.com/submissions

